\title{
INCLUSIÓN SOCIAL \\ Y AUTODETERMINACIÓN: LOS RETOS \\ EN LA CALIDAD DE VIDA \\ DE LOS JÓVENES CON AUTISMO \\ Y DISCAPACIDAD INTELECTUAL
}

\section{Social inclusion and self-determination: the challenges in the quality of life of youth with autism and intellectual disability}

\author{
M. ${ }^{a}$ Lucía Morán SuÁReZ \\ moranlucia@uniovi.es \\ Laura E. Gómez SÁNCHEZ \\ Facultad de Psicología. Universidad de Oviedo \\ M. ángeles Alcedo Rodríguez \\ Facultad de Psicología. Universidad de Oviedo
}

Facultad de Psicología. Universidad de Oviedo. Plaza Feijoo, s/n. 33003 Oviedo

Recepción: 11 de septiembre de 2018

Aceptación definitiva: 30 de enero de 2019

Resumen: Entre los colectivos que se han beneficiado en menor medida de los progresos en la aplicación del concepto de calidad de vida se encuentra el de niños y jóvenes con autismo. El objetivo de este estudio es presentar la nueva Escala KidsLife-TEA y los primeros resultados obtenidos tras su aplicación. La muestra estuvo compuesta por 420 personas con discapacidad intelectual y autismo de entre 4 y 21 años que reciben apoyos en 78 organizaciones españolas. Se utilizó la Escala KidsLife-TEA, que evalúa resultados personales en calidad de vida de jóvenes con autismo y discapacidad intelectual. Las mayores puntuaciones se obtuvieron en bienestar material y desarrollo personal, mientras que las más bajas se dieron en inclusión social y autodeterminación. Se discuten los hallazgos del estudio y se apuntan líneas futuras para mejorar la calidad de vida de este colectivo. 
INCLUSIÓN SOCIAL Y AUTODETERMINACIÓN: LOS RETOS EN LA CALIDAD DE VIDA DE LOS JÓVENES CON AUTISMO Y DISCAPACIDAD INTELECTUAL

M. ${ }^{a}$ LUCÍA MORÁN SUÁREZ, LAURA E. GÓMEZ SÁNCHEZ Y M. ${ }^{a}$ ÁNGELES ALCEDO RODRÍGUEZ

Palabras clave: calidad de vida; autismo; discapacidad intelectual; infancia; adolescencia.

Aвstract: Among the groups which have had least benefit from the progress in applicating the quality of life concept are children and youth with autism. The aim of this study is presenting the new scale KidsLife-ASD and the first results after its application. The sample was composed of 420 people with intellectual disability and autism between 4 and 21 years old who were attending 78 Spanish organizations. The KidsLife-AsD scale was used, which evaluates quality of life personal outcomes of youth with autism and intellectual disabilities. The highest results were obtained in material wellbeing and personal development, while the lowest scores were shown in social inclusion and self-determination. The results from the study are discussed and future lines are proposed to enhance the quality of life of this group.

KEY WORDs: quality of life; autism; intellectual disability; childhood; adolescence.

1. Inclusión social y autodeterminación: los retos en la calidad de vida de los jóvenes con autismo y discapacidad intelectual

4 L CONCEPTO DE CALIDAD DE VIDA ha adquirido en los últimos años un papel central no solo a la hora de guiar y contrastar la eficacia de las prácticas profesionales, sino también en la redefinición de las estrategias organizacionales y las políticas sociales en los ámbitos educativo, sanitario y de servicios sociales (Bigby, Knox, Beadle-Brown y Bould, 2014; Schalock, Verdugo, Gómez y Reinders, 2016; van Loon et al., 2013). En la atención a personas con discapacidad intelectual (DI), la introducción del enfoque biopsicosocial -que asume que en la discapacidad no solo existen limitaciones, sino también fortalezas, y que el funcionamiento del individuo en interacción con el entorno que le rodea mejorará si se le ofrecen los apoyos adecuados (Luckasson et al., 2002; Schalock et al., 2011)- ha propiciado el uso del modelo de calidad de vida como marco de referencia para la provisión de apoyos y servicios (Verdugo, Gómez y Navas, 2013; Schalock y Verdugo, 2012).

Existen numerosos modelos de calidad de vida, siendo el más extendido en el contexto español entre los proveedores de servicios (Arias et al., 2018; Carbó-Carreté, Guàrdia-Olmos y Giné, 2015; Fernández, Verdugo, Gómez, Aguayo y Arias, 2018; Gómez, Arias, Verdugo, Tassé y Brown, 2015; Gómez, Alcedo, Arias et al., 2016; Morán, Gómez y Alcedo, 2015; Verdugo, Gómez, Arias, Navas y Schalock, 2014) y el que mayor cantidad de evidencias empíricas acumula (Claes, van Hove, Vandevelde, van Loon y Schalock, 2012; Gómez, Verdugo, Arias y Arias, 2011; Jenaro et al., 2005; van Loon, Claes y Schalock, 2014; Wang et al., 2010) el propuesto por Schalock y Verdugo (2002/2003, 2012), quienes definen la calidad de vida como un estado deseado de bienestar personal que: (a) es multidimensional; (b) tiene propiedades universales y ligadas a la cultura; (c) engloba componentes objetivos y subjetivos; y (d) está influenciado por características personales y factores ambientales (Schalock,

(C) Ediciones Universidad de Salamanca / CC BY-NC-ND

Siglo Cero, vol. 50 (3), n. ${ }^{\circ}$ 271, 2019, julio-septiembre, pp. 29-46 
Keith, Verdugo y Gómez, 2010). Concretamente, en este modelo se distinguen ocho dimensiones esenciales de calidad de vida que son importantes para todas las personas: bienestar emocional, bienestar físico, bienestar material, relaciones interpersonales, desarrollo personal, autodeterminación, inclusión social y derechos.

Si bien se han producido importantes avances en la operativización y aplicación del concepto de calidad de vida en el ámbito de la discapacidad, los niños y jóvenes con Di y Trastornos del Espectro del Autismo (TEA) conforman uno de los grupos que se han beneficiado en menor medida de estos cambios. Tanto es así que, hasta la fecha, no se encontraban en la literatura especializada instrumentos que permitiesen un abordaje específico de la evaluación de calidad de vida en jóvenes con TEA con suficientes garantías psicométricas (Gómez, Morán, Alcedo, Arias et al., 2018; Knüppel, Jakobsen, Lauritsen y Telléus, 2018; van Heijst y Geurts, 2015; Tavernor, Barron, Rodgers y McConachie, 2013), a pesar de que: (a) ha aumentado significativamente la prevalencia de los TEA en las últimas décadas (Idring et al., 2015; Morales-Hidalgo, Roige-Castellvi, Hernández-Martínez, Voltas y Canals, 2018); (b) se han apuntado menores niveles de calidad de vida en niños con TEA en comparación con niños sin discapacidad (Clark, Magill-Evans y Koning, 2015; Chiang y Wineman, 2014; de Vries y Geurts, 2015; Ikeda, Hinckson y Krägeloh, 2014; Kuhlthau et al., 2013) o con enfermedades crónicas (Cottenceau et al., 2012); y (c) la relevancia del tema se ha visto reflejada en la reciente aprobación de la primera Estrategia Nacional para la mejora de la calidad de vida de las personas con TEA (Ministerio de Sanidad, Servicios Sociales e Igualdad, 2015). Así pues, los instrumentos para la evaluación de la calidad de vida con suficientes garantías de validez y fiabilidad son no ya recomendables, sino necesarios e indispensables (Ley de Promoción de la Autonomía Personal y Atención a las Personas en Situación de Dependencia, $B O E$ de 15 de diciembre de 2006: 44154).

En esta línea, dado que las intervenciones dirigidas a la mejora de la calidad de vida deben basarse en evidencias, se han desarrollado y validado en el contexto español diversos instrumentos de calidad de vida a partir del modelo propuesto por Schalock y Verdugo (2002/2003), tales como la Escala GENCAT (Verdugo, Arias, Gómez y Schalock, 2010), dirigida a usuarios de servicios sociales; la Escala INICO-FEAPs (Gómez, Verdugo y Arias, 2015) para adultos con Di; la Escala San Martín (Verdugo, Gómez, Arias y Schalock, 2014) para adultos con necesidades de apoyo significativas; la Escala de Calidad de vida para personas con discapacidad que envejecen (Alcedo, Aguado, Arias, González y Rozada, 2008) y la Escala cavidace (Fernández et al., 2018) para adultos con daño cerebral. En el caso de los más jóvenes, se desarrollaron la CVI-Cvip (Sabeh, Verdugo, Prieto y Contini, 2009) y la ccva (Gómez-Vela y Verdugo, 2009), destinadas, respectivamente, a niños y adolescentes. Más recientemente, superando las limitaciones psicométricas de las dos anteriores, para la evaluación de niños y jóvenes con DI y necesidades de apoyos significativas se han desarrollado la Escala KidsLife (Gómez, Alcedo, Arias et al., 2016) y su versión para personas con síndrome de Down (Gómez et al., 2017).

Ante la demanda de los profesionales y organizaciones que atienden a personas con autismo de contar con instrumentos que tengan en cuenta las características y necesidades específicas de las personas con TEA, se ha desarrollado la Escala KidsLife-TEA 
(Gómez, Morán, Alcedo, Verdugo et al., 2018), siendo esta la primera escala existente para evaluar resultados personales relacionados con calidad de vida de forma específica en niños y adolescentes con autismo y DI. Por este motivo, el objetivo del presente estudio consiste en explorar los resultados de calidad de vida obtenidos tras la aplicación de la Escala KidsLife para personas con TEA a una amplia muestra española, en aras de establecer cuáles son las dimensiones que siguen suponiendo un reto en nuestros días por ser susceptibles de mejoras.

\section{Método}

\subsection{Participantes}

La muestra de estudio estuvo compuesta por 420 personas con DI y TEA. Los criterios de inclusión fueron: (a) presentar de forma conjunta TEA y DI; (b) tener entre 4 y 21 años, y (c) estar recibiendo apoyos y servicios en una organización que tenga entre sus objetivos la mejora de la calidad de vida. El único criterio de exclusión fue encontrarse fuera del sistema educativo.

El rango de edad osciló entre 4 y 21 años, con una media de 11.97 años $(D T=4.73)$. El $79.3 \%$ de la muestra estaba compuesta por varones $(n=333)$ frente a un $20.7 \%$ de mujeres $(n=87)$. El $12.6 \%$ tenía un nivel de Di leve, el $37.1 \%$ moderado, el $44.3 \%$ severo y el $6 \%$ profundo. Respecto al nivel de necesidades de apoyos, el $5.2 \%$ presentaba un nivel limitado, el $13.3 \%$ intermitente, el $44.5 \%$ extenso y el $36.9 \%$ generalizado. La mayor parte presentó un Grado III de Dependencia (47.9\%), seguido de Grado II (36.4\%) y Grado I (15\%). Las condiciones asociadas con más frecuencia al TEA fueron los problemas de comportamiento (16.2\%), la discapacidad física $(8.3 \%)$, la epilepsia $(6.9 \%)$, los problemas de salud mental o trastornos emocionales $(6.7 \%)$, la discapacidad visual (3.3\%) y el síndrome de Down (3.3\%). Una amplia mayoría de participantes contaba con familia (98.6\%) y residían en el domicilio familiar $(86.7 \%)$. La mayor parte estaban escolarizados en la modalidad de educación especial $(65.2 \%)$, seguidos de educación ordinaria $(22.1 \%$ ) y combinada (12.6\%).

Las 420 escalas fueron cumplimentadas por 237 informadores pertenecientes a 78 entidades u organizaciones de toda España. La mayoría eran profesionales de atención directa $(51.5 \%)$, sobre todo profesores y psicólogos. Los restantes eran padres o madres $(48.5 \%)$. El $82.4 \%$ sobre el total de informantes eran mujeres, principalmente madres (45.8\%). La media de los años de relación con la persona evaluada era de 5.36 años $(D T=4.88)$ y la frecuencia del contacto era diaria en el $83.6 \%$ de los casos. De las entidades participantes, el $52.6 \%$ eran de tipo concertado o privado $(37.2 \%)$, predominando servicios de tipo educativo $(74.4 \%)$, seguidas de entidades de tipo social $(23.1 \%)$ y sanitario $(2.6 \%)$.

\subsection{Instrumento}

Se utilizó la Escala KidsLife tea (Gómez, Morán, Alcedo, Verdugo et al., 2018) que evalúa resultados personales de calidad de vida en niños y jóvenes entre 4 y 21

(C) Ediciones Universidad de Salamanca / CC BY-NC-ND

Siglo Cero, vol. 50 (3), n. ${ }^{\circ}$ 271, 2019, julio-septiembre, pp. 29-46 
años con TEA y DI. Cuenta con 96 ítems divididos en las ocho dimensiones de calidad de vida del modelo de Schalock y Verdugo (2002/2003). Cada dimensión está formada por 12 ítems con cuatro opciones de frecuencia (nunca, a veces, frecuentemente y siempre). La escala también incluye una sección de datos sociodemográficos de la persona evaluada, del evaluador principal y de la organización en la que la persona recibe apoyos y servicios. La evaluación es realizada por un informador o informadores que conocen bien a la persona, desde hace al menos seis meses y con oportunidades de observarla en diferentes ambientes durante períodos prolongados de tiempo (p. ej., familiares, profesionales y proveedores de apoyos). El tiempo medio de cumplimentación es de 30 minutos.

Este instrumento constituye una adaptación para personas con TEA de la Escala KidsLife (Gómez, Alcedo, Verdugo et al., 2016), que permite evaluar calidad de vida de niños y jóvenes con DI y grandes necesidades de apoyo. Para su desarrollo, se partió de la conceptualización y aplicación de la versión piloto de la Escala KidsLife original, conformada por 156 ítems (20 ítems por dimensión, a excepción de inclusión social, que contaba con 16 ítems). Dicha versión piloto fue desarrollada mediante una exhaustiva revisión de literatura científica, la selección de ítems provenientes de diversas escalas dirigidas a personas con discapacidad y la propuesta de nuevos ítems, la realización de un estudio Delphi de tres rondas en el que participaron 14 jueces expertos en calidad de vida y en el ámbito de la DI (Gómez et al., 2014) y la selección final de ítems por parte del equipo de investigación.

La posterior adaptación de la versión piloto de la escala al colectivo de personas con TEA implicó la combinación de análisis cuantitativos (obtención de medias e índices de homogeneidad corregida de los ítems) y cualitativos (contenido adecuado para TEA) con el fin de reducir el número de ítems y seleccionar los más idóneos que conforman la versión final de la Escala KidsLife-TEA. La escala fue validada con la muestra del presente estudio $(N=420)$. Con esta muestra, la escala total obtuvo un coeficiente alfa de Cronbach de .95, mientras que en las dimensiones (Figura 1) oscilaron entre .74 (bienestar físico) y .88 (desarrollo personal). En la Tabla 1 pueden observarse los coeficientes de Spearman-Brown $\left(r_{x x}\right)$, los coeficientes lambda de Guttman $\left(\lambda_{1}, \lambda_{3}\right.$ y $\left.\lambda_{2}\right)$, los coeficientes de alfa de Cronbach $(\alpha)$, los coeficientes theta ordinal $(\theta$ ordinal) y los coeficientes omega $(\Omega)$ por dimensiones. Información más amplia sobre el proceso de validación de la escala puede consultarse en Gómez et al. (Gómez, Morán, Alcedo, Arias et al., 2018).

La Escala KidsLife-TEA cuenta con baremos para tres grupos de edad (4-8, 9-14 y 15-21 años) y proporciona puntuaciones estándar $(M=10 ; D T=3)$ y percentiles para las ocho dimensiones de calidad de vida, así como un índice general de calidad de vida $(M=100 ; D T=15)$ con su correspondiente percentil. Todas estas puntuaciones pueden ilustrarse en el perfil de calidad de vida. La versión electrónica de la escala está disponible gratuitamente en la web del INICO (http://sid.usal.es/27385/8-1). Existe, además, una versión en inglés de la escala y varias adaptaciones para su aplicación en otros países (p. ej., Argentina, Bélgica, Chile, Colombia, Estados Unidos, Japón, Holanda, Italia, México, Noruega, Reino Unido, entre otros). 
INCLUSIÓN SOCIAL Y AUTODETERMINACIÓN: LOS RETOS EN LA CALIDAD DE VIDA DE LOS JÓVENES CON AUTISMO Y DISCAPACIDAD INTELECTUAL

M. ${ }^{a}$ LUCÍA MORÁN SUÁREZ, LAURA E. GÓMEZ SÁNCHEZ Y M. ${ }^{a}$ ÁNGELES ALCEDO RODRÍGUEZ

\section{FIGURA 1. Consistencia interna (alpha de Cronbach) de las dimensiones}

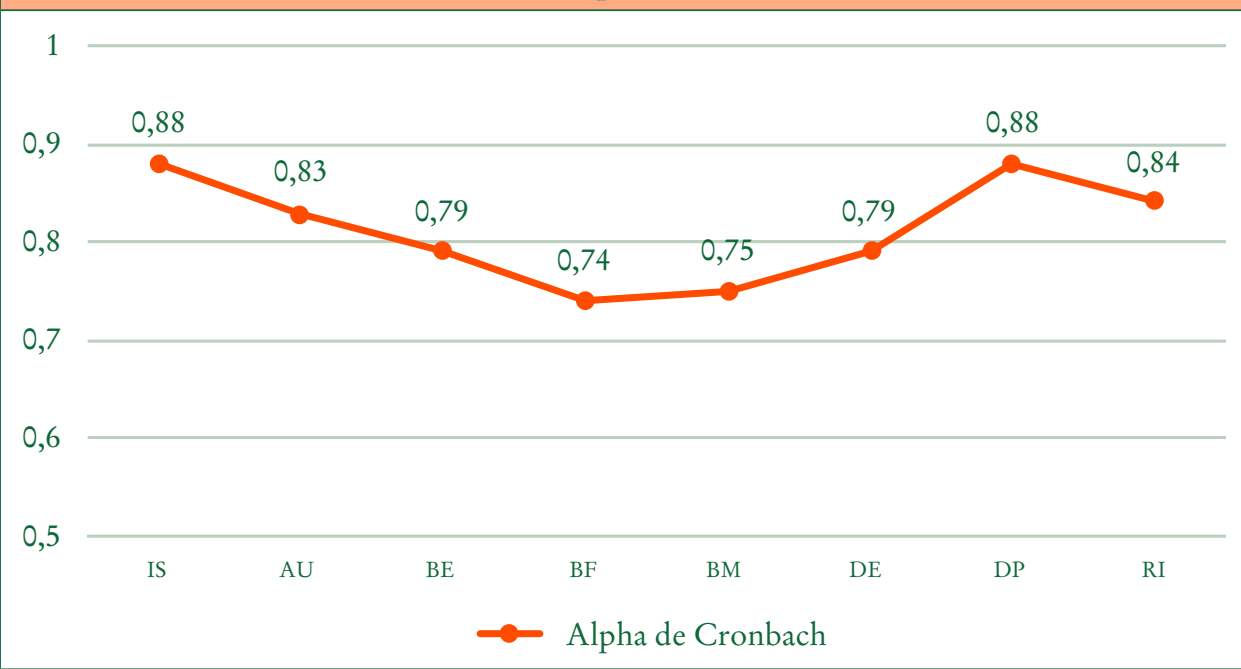

Nota. IS = inclusión social; $\mathrm{AU}=$ autodeterminación; $\mathrm{BE}=$ bienestar emocional; $\mathrm{BF}=$ bienestar físico; $\mathrm{BM}=$ bienestar material; $\mathrm{DE}=$ derechos; social; $\mathrm{AU}=$ autodeterminación; $\mathrm{BE}=$ bienestar emocional.

\section{TABLA 1. Coeficientes de consistencia interna por dimensiones}

\begin{tabular}{|l|c|c|c|c|c|c|}
\hline Dimensiones & $\mathrm{r}_{\mathrm{xx}}$ & $\lambda_{1}$ & $\lambda_{3}=\alpha$ & $\lambda_{2}$ & $\theta$ ordinal & $\Omega$ \\
\hline Inclusión social & .89 & .81 & .88 & .88 & .91 & .87 \\
\hline Autodeterminación & .77 & .77 & .83 & .84 & .88 & .86 \\
\hline Bienestar emocional & .75 & .73 & .79 & .80 & .86 & .83 \\
\hline Bienestar físico & .73 & .68 & .74 & .76 & .83 & .74 \\
\hline Bienestar material & .72 & .70 & .75 & .76 & .86 & .77 \\
\hline Derechos & .76 & .72 & .79 & .81 & .89 & .83 \\
\hline Desarrollo personal & .86 & .81 & .88 & .89 & .93 & .90 \\
\hline Relaciones interpersonales & .80 & .77 & .84 & .85 & .89 & .87 \\
\hline
\end{tabular}

Nota. $\mathrm{r}_{\mathrm{xx}}=$ coeficientes de Spearman-Brown; $\lambda_{1}, \lambda_{2}, \lambda_{3}=$ coeficientes lambda de Guttman; $\alpha=$ coeficientes alpha de Cronbach; $\theta$ ordinal = coeficientes theta ordinal; $\Omega=$ coeficientes omega.

\subsection{Procedimiento}

Se divulgó información sobre los objetivos del estudio y cómo participar en el mismo a través de congresos científicos, cursos, seminarios, redes sociales (Facebook, Twitter, ResearchGate) y páginas web (Plena inclusión e INICO). A su vez, el equipo de investigación llevó a cabo una exhaustiva búsqueda en internet de organizaciones, centros y colegios que prestan apoyos y servicios a personas con DI y autismo. Se

(C) Ediciones Universidad de Salamanca / CC BY-NC-ND

Siglo Cero, vol. 50 (3), n. ${ }^{\circ}$ 271, 2019, julio-septiembre, pp. 29-46 
realizó un envío masivo de correos electrónicos solicitando la participación de las organizaciones (y llamadas a las que no tenían disponible una dirección electrónica de contacto).

A aquellos centros y organizaciones que respondieron favorablemente a la invitación, se les remitió el enlace a una encuesta online para inscribirse en el estudio en la que se les solicitaban sus datos de contacto y los de la persona encargada de coordinar el proceso de evaluación en cada centro. Una vez inscritos los centros en el estudio, se les envió a los coordinadores el material necesario para cumplimentar las escalas: (a) un enlace para acceder a la escala, al manual de instrucciones y al consentimiento informado; y (b) los datos de contacto del equipo de investigación para solventar dudas o hacer comentarios (mediante correo electrónico, llamadas telefónicas o reuniones presenciales en los centros).

Las escalas podían ser cumplimentadas en versión electrónica o en papel. En este último caso, las escalas fueron enviadas por correo postal al equipo de investigación con el fin de añadir los resultados a una base de datos y llevar a cabo los análisis estadísticos pertinentes. Durante el proceso de administración de escalas, no se recogieron datos personales que facilitasen reconocer a la persona evaluada. En su lugar se utilizaron códigos de identificación (p. ej., seudónimos) que eran anónimos para las investigadoras. Tales códigos de identificación permitieron devolver a las organizaciones los resultados de las evaluaciones con el fin de que fueran útiles en el diseño y evaluación de planificaciones centradas en la persona.

Los datos fueron analizados mediante el paquete estadístico spss 24.0. La investigación fue aprobada por el Comité Ético de la Universidad de Oviedo y cumplió con los principios de la declaración de Helsinki.

\section{Resultados}

La distribución de las puntuaciones obtenidas con la Escala KidsLife-Tea (Tabla 2) presentó una ligera asimetría negativa (asimetría $=-.193$; curtosis $=-.347$ ). Teniendo en cuenta que la puntuación mínima posible de la escala es 96 y la máxima 384, las puntuaciones variaron entre 177 y 362. La media se sitúo en $289.62(D T=34.86)$ y la mediana en 291. Tanto la media como la mediana sobrepasaron ampliamente el punto medio teórico de la escala (Punto Medio Teórico $=192$ ), valor que ya fue superado por el percentil 25. Asimismo, la puntuación alcanzada con mayor frecuencia por los participantes fue 273.

\begin{tabular}{|c|c|c|c|c|c|c|c|c|c|}
\hline \multicolumn{10}{|c|}{$\begin{array}{l}\text { TABLA 2. Estadísticos descriptivos de las puntuaciones en la escala } \\
\text { y sus dimensiones }\end{array}$} \\
\hline Descriptivos & $\mathrm{CV}$ & IS & $\mathrm{AU}$ & $\mathrm{BE}$ & BF & BM & $\mathrm{DE}$ & DP & RI \\
\hline Media & 289.62 & 28.33 & 28.78 & 38.68 & 38.88 & 40.56 & 38.47 & 39.90 & 36.00 \\
\hline E. T. Media & 1.70 & .34 & .32 & .27 & .28 & .24 & .27 & .28 & .31 \\
\hline $\mathrm{N}$ válido & 420 & 420 & 420 & 420 & 420 & 420 & 420 & 420 & 420 \\
\hline
\end{tabular}


INCLUSIÓN SOCIAL Y AUTODETERMINACIÓN: LOS RETOS EN LA CALIDAD DE VIDA DE LOS JÓVENES CON AUTISMO Y DISCAPACIDAD INTELECTUAL

M. ${ }^{a}$ LUCÍA MORÁN SUÁREZ, LAURA E. GÓMEZ SÁNCHEZ Y M. ${ }^{a}$ ÁNGELES ALCEDO RODRÍGUEZ

\begin{tabular}{|c|c|c|c|c|c|c|c|c|c|}
\hline \multicolumn{10}{|c|}{$\begin{array}{l}\text { TABLA 2. Estadísticos descriptivos de las puntuaciones en la escala } \\
\text { y sus dimensiones (cont.) }\end{array}$} \\
\hline Descriptivos & $\mathrm{CV}$ & \begin{tabular}{|l|} 
IS \\
\end{tabular} & $\mathrm{AU}$ & \begin{tabular}{|c|}
$\mathrm{BE}$ \\
\end{tabular} & \begin{tabular}{|l|}
$\mathrm{BF}$ \\
\end{tabular} & BM & \begin{tabular}{|l|}
$\mathrm{DE}$ \\
\end{tabular} & \begin{tabular}{|l|} 
DP \\
\end{tabular} & RI \\
\hline Mediana & 291.00 & 28.00 & 29.00 & 39.00 & 40.00 & 41.00 & 39.00 & 40.00 & 36.50 \\
\hline Moda & 273.00 & 28.00 & 27.00 & 43.00 & 43.00 & 42.00 & 37.00 & 48.00 & 37.00 \\
\hline Mínimo & 177.00 & 14.00 & 12.00 & 15.00 & 21.00 & 23.00 & 23.00 & 21.00 & 12.00 \\
\hline Máximo & 362.00 & 48.00 & 47.00 & 48.00 & 48.00 & 48.00 & 48.00 & 48.00 & 48.00 \\
\hline Pto. Medio Teórico & 192 & 24 & 24 & 24 & 24 & 24 & 24 & 24 & 24 \\
\hline Varianza & 1215.41 & 49.53 & 42.24 & 31.22 & 33.35 & 24.32 & 29.84 & 32.72 & 40.27 \\
\hline DT & 34.86 & 7.04 & 6.50 & 5.59 & 5.77 & 4.93 & 5.46 & 5.72 & 6.35 \\
\hline Rango & 185 & 34 & 35 & 33 & 27 & 25 & 25 & 27 & 36 \\
\hline \multicolumn{10}{|l|}{ Percentiles } \\
\hline 25 & 264.25 & 23.00 & 24.00 & 35.00 & 35.00 & 38.00 & 35.00 & 36.00 & 31.00 \\
\hline 50 & 291.00 & 28.00 & 29.00 & 39.00 & 40.00 & 41.00 & 39.00 & 40.00 & 36.50 \\
\hline 75 & 315.00 & 32.00 & 33.00 & 43.00 & 43.00 & 44.00 & 43.00 & 45.00 & 41.00 \\
\hline 100 & 362 & 48 & 47 & 48 & 48 & 48 & 48 & 48 & 48 \\
\hline Asimetría & -.193 & .550 & .178 & -.710 & -.474 & -.565 & -.308 & -.468 & -.353 \\
\hline Curtosis & -.35 & -.12 & -.45 & .35 & -.40 & -.06 & -.56 & -.34 & .10 \\
\hline
\end{tabular}

Nota $. \mathrm{Cv}=$ calidad de vida; $\mathrm{is}=$ inclusión social; $\mathrm{AU}=$ autodeterminación; $\mathrm{BE}=$ bienestar emocional; $\mathrm{BF}=$ bienestar físico; $\mathrm{BM}=$ bienestar material. $\mathrm{DE}=$ derechos; $\mathrm{DP}=$ desarrollo personal; $\mathrm{RI}=$ relaciones interpersonales.

El análisis detallado de las respuestas a los ítems en cada una de las ocho dimensiones de calidad de vida (teniendo en cuenta que cada una cuenta con 12 ítems, siendo la puntuación mínima posible de 12 y la máxima 48) reveló que en todas ellas la media superó el punto medio teórico de 24. Las puntuaciones medias más altas se obtuvieron en las dimensiones bienestar material y desarrollo personal. Las puntuaciones más bajas, en cambio, se observaron en inclusión social y autodeterminación (Figura 2).

Si atendemos a las respuestas más positivas ("siempre") en las dimensiones bienestar material y desarrollo personal, observamos como la mayoría de participantes cuenta con sus propios materiales para entretenerse (68.8\%); se repone o repara su material cuando está deteriorado $(68.6 \%)$; cuenta con las ayudas técnicas apropiadas para maximizar su autonomía (68.6\%); dispone de los bienes materiales que necesita (67.4\%); cuenta con material didáctico adaptado (65.2\%); recibe apoyos y atención individualizados (65\%); tiene ropa adecuada a su gusto y edad $(64.3 \%)$; vive en un lugar adaptado a sus características físicas sensoriales e intelectuales (61.7\%); tiene fortalezas que son tenidas en cuenta cuando se plantean nuevos objetivos (59\%); acuden a un centro educativo adaptado a sus características físicas, sensoriales e intelectuales (59\%); le gustan las cosas que tiene (p. ej., juguetes, materiales escolares, ropa) $(55.7 \%)$; disfruta de medidas específicas para mantener sus capacidades y habilidades $(54.3 \%)$, y de instrucciones y modelos para aprender cosas nuevas $(50.7 \%)$. Estos resultados se muestran en las Figuras 3 y 4 .

(C) Ediciones Universidad de Salamanca / CC BY-NC-ND

Siglo Cero, vol. 50 (3), n. ${ }^{\circ} 271,2019$, julio-septiembre, pp. 29-46 

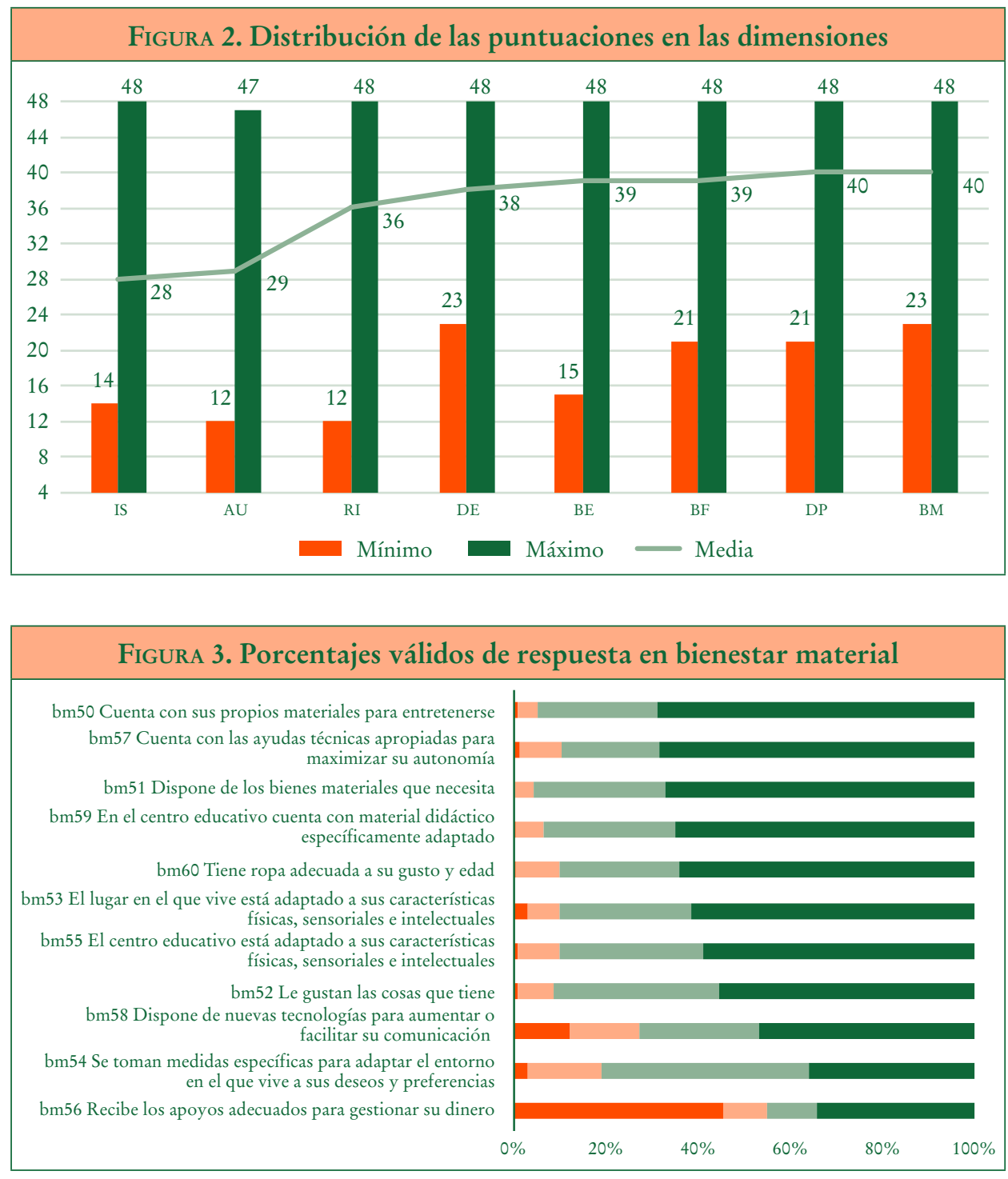

En contraposición, en las dimensiones inclusión social y autodeterminación observamos entre las respuestas más negativas de los informadores ("nunca") que una gran parte de los participantes no participa en la elaboración de su plan individual de apoyos $(65.7 \%)$ ni en grupos naturales de su comunidad (55\%). Casi la mitad no decora la habitación a su gusto $(47.6 \%)$ ni elige la ropa que se quiere poner $(46.7 \%)$ ni participa en actividades fuera del centro con personas ajenas a su contexto de apoyos $(41 \%)$. Una tercera parte $(33.8 \%)$ no elige con quién pasar su tiempo libre y una

(C) Ediciones Universidad de Salamanca / CC BY-NC-ND

Siglo Cero, vol. 50 (3), n. ${ }^{\circ}$ 271, 2019, julio-septiembre, pp. 29-46

$$
-37-
$$




\section{FiguRa 4. Porcentajes válidos de respuesta en desarrollo personal}

\begin{tabular}{|r|r|}
\hline dp81 Recibe apoyos y atención individualizados & \\
dp84 Se tienen en cuenta sus fortalezas cuando se plantean nuevos objetivos & \\
dp80 Se toman medidas específicas para mantener sus capacidades y habilidades & \\
dp78 Se le proporcionan instrucciones y modelos para aprender cosas nuevas & \\
dp74 La estimulación de su desarrollo se lleva a cabo respetando sus ritmos y preferencias & \\
dp75 Aprende cosas que le hacen ser más independiente & \\
dp83 Las personas que le proporcionan apoyos reciben formación sobre los métodos & \\
dp77 Las actividades que realiza le permiten el aprendizaje de nuevas habilidades & \\
dp73 Tiene un programa de actividades con cosas que le gustan & \\
dp79 Tiene oportunidades para demostrar sus habilidades & \\
dp82 Se le ayuda a planificar las actividades que quiere realizar cuando lo necesita & \\
dp76 Se le enseñan actividades instrumentales de la vida diaria & \\
\hline
\end{tabular}

cuarta parte no participa en actividades sociales fuera del lugar donde recibe servicios o apoyos (25.2\%). La opinión de una quinta parte de los participantes (19\%) no es tenida en cuenta cuando se realizan cambios en los centros en los que reciben apoyos. Además, un número considerable no participa en actividades de ocio y cultura en entornos comunitarios $(17.9 \%)$, no interactúa con personas ajenas a su contexto de

\section{FiguRA 5. Porcentajes válidos de respuesta en inclusión social}

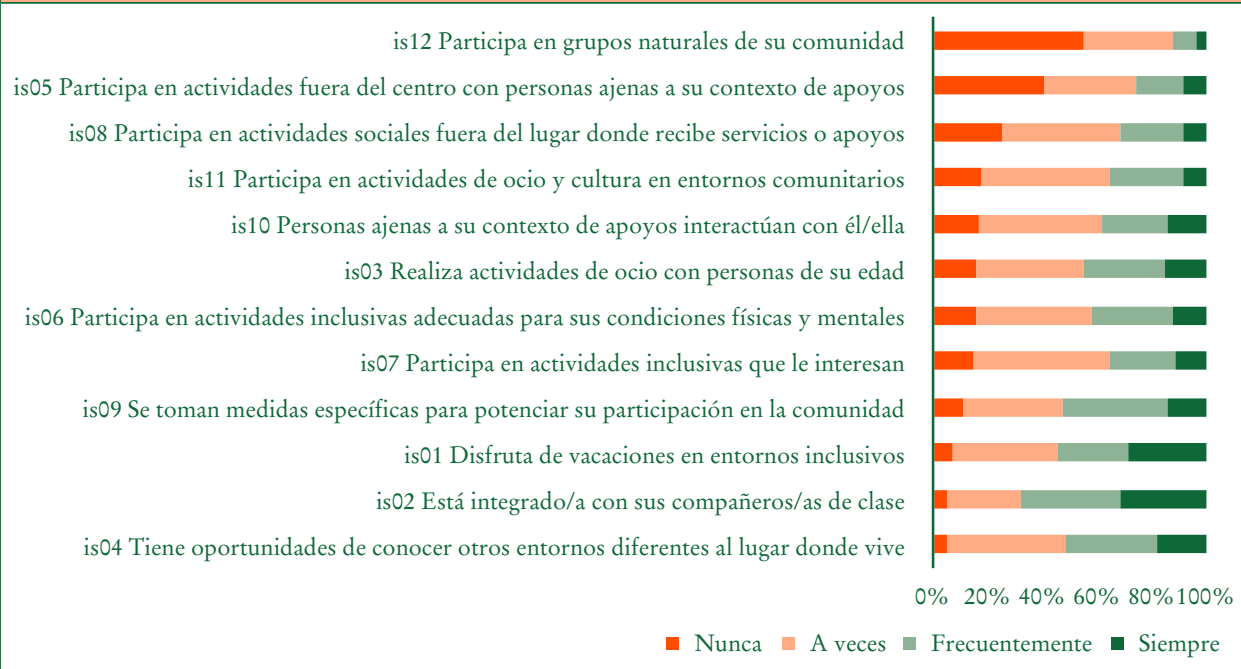

(C) Ediciones Universidad de Salamanca / CC BY-NC-ND

Siglo Cero, vol. 50 (3), n. ${ }^{\circ}$ 271, 2019, julio-septiembre, pp. 29-46

$$
-38-
$$


apoyos (17.1\%), no realiza actividades de ocio con personas de su edad (16\%), no participa en actividades inclusivas adecuadas para sus condiciones físicas y mentales $(15.5 \%)$ ni en actividades inclusivas que le interesen (15.2\%). En las Figuras 5 y 6 pueden observarse estos resultados.

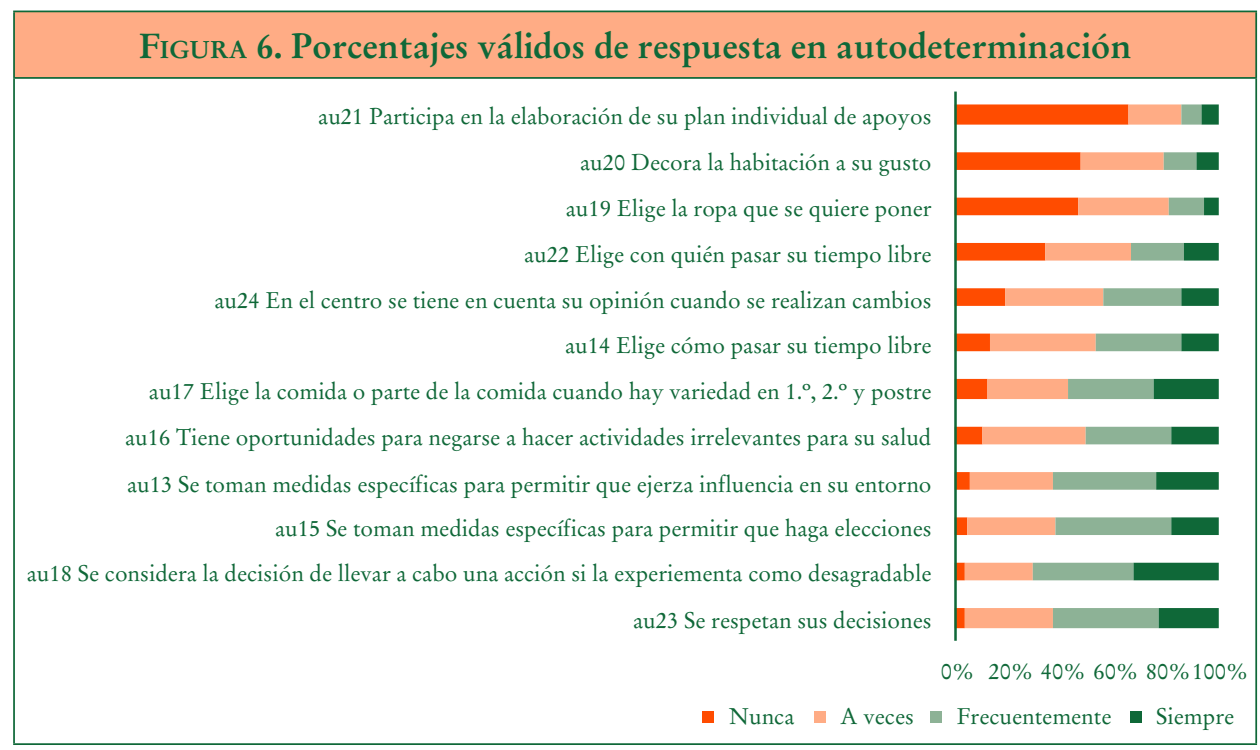

\section{Discusión}

Los principales objetivos de este trabajo se centran en presentar la nueva Escala KidsLife-TEA, el primer instrumento disponible para evaluar calidad de vida específicamente en niños y jóvenes con TEA y DI, con el fin de orientar los apoyos, las intervenciones y las estrategias dirigidas a la mejora de la calidad. Del mismo modo, se ha tratado de explorar de forma preliminar los resultados personales relacionados con calidad de vida obtenidos con la escala por una amplia muestra española.

En general, podemos concluir que las puntuaciones en calidad de vida de niños y jóvenes con TEA y Di son positivas. Atendiendo a los resultados medios obtenidos en cada una de las dimensiones, las mayores fortalezas se dieron en bienestar material y desarrollo personal. Vemos, pues, como las áreas en las que se obtienen resultados más positivos tienen que ver con el fomento del bienestar material, mediante la disponibilidad de bienes materiales (p. ej., ayudas técnicas, material didáctico), aspecto estrechamente relacionado con el paradigma de la rehabilitación de la discapacidad que ha imperado en España en las últimas décadas. Estos excelentes resultados en bienestar material son congruentes con lo observado en el estudio de la calidad de vida de otros colectivos con discapacidad o en riesgo de exclusión social que son usuarios de servicios sociales en nuestro país (Arias, Gómez, Verdugo

(C) Ediciones Universidad de Salamanca / CC BY-NC-ND

Siglo Cero, vol. 50 (3), n. ${ }^{\circ}$ 271, 2019, julio-septiembre, pp. 29-46 
y Navas, 2010; Gómez, 2010, 2014; Gómez, Arias, Verdugo y Navas, 2012; Santamaría et al., 2012).

La segunda área con mejores resultados fue el desarrollo personal. Destaca que en el presente estudio, para un gran porcentaje de la muestra, los apoyos y la atención están adaptados a sus características individuales, se tienen en cuenta sus fortalezas y se toman medidas para mantener sus habilidades y que aprendan cosas nuevas, lo que supone un importante avance hacia un enfoque centrado en la persona, en el que se valore lo que es importante para la misma y lo que tiene impacto en su vida (Schalock, 2015). No obstante, los ítems con resultados más positivos continúan siendo los que implican a terceras personas tales como profesionales, familiares y organizaciones, y no tanto a la persona con discapacidad de forma activa. Del mismo modo, resulta llamativo que se obtengan estos resultados tan positivos en desarrollo personal cuando en estudios precedentes en adultos con DI esta dimensión suele destacarse entre las dimensiones con resultados más limitados (Gómez, 2010, 2014; Gómez et al., 2011; Morán, Gómez y Alcedo, 2018). Quizás estas discrepancias podrían deberse al hecho de que todos los participantes de este trabajo se encuentran en el sistema educativo y que por ello estén recibiendo más y mejores apoyos con respecto a los indicadores típicos de esta dimensión (formación, habilidades, competencias), mientras las puntuaciones de los adultos con DI pueden verse afectadas por las limitaciones e incluso la inexistencia en él de programas de transición a la vida adulta y envejecimiento activo que den continuidad a las competencias trabajadas y los objetivos planteados durante la etapa educativa.

Por otro lado, los respondientes informaron de mayores dificultades en las dimensiones inclusión social y autodeterminación, y de forma respectiva en los ítems relacionados con participar en grupos naturales de su comunidad o en actividades fuera del centro (inclusión social) y con la participación en la elaboración de su plan individual de apoyos y en la decoración de su habitación (autodeterminación). Estos resultados resultan congruentes con los obtenidos en estudios precedentes sobre este y otros colectivos (Fernández et al., 2015; Morán et al., 2018; Gómez, 2010, 2014). Así, las áreas prioritarias en las que se dieron las mayores limitaciones fueron aquellas que tienen como protagonista a la propia persona con TEA, tales como la participación en la vida comunitaria y la toma de decisiones. Frente a ello, resulta fundamental promover la asistencia a actividades sociales y capacitar a la persona con TEA para tener más control sobre su propia vida y mejorar sus posibilidades de elección, especialmente en las etapas de la infancia y adolescencia (Verdugo et al., 2013; White, Flanagan y Nadig, 2018). Para ello, ha de tenerse en cuenta que tales aspectos no solo dependen de la propia persona con discapacidad, sino también de las oportunidades y apoyos que brinda el entorno (Burgess y Gutstein, 2007; Chou, Wehmeyer, Palmer y Lee, 2016; Peralta y Arellano, 2014). En este sentido, destaca la necesidad de desarrollar estudios específicos que permitan evaluar y fomentar la autodeterminación de las personas con TEA y utilizar instrumentos desarrollados específicamente para evaluar la autodeterminación (Mumbardó-Adam, Guàrdia-Olmos, Giné, Shogren y Vicente, 2018; Shogren et al., 2018; Vicente, Guillén, Gómez, Ibáñez y Sánchez, 2018) no solo para contrastar y profundizar en los datos obtenidos, sino también para comprobar su grado de adecuación y utilidad en este colectivo 
Como toda investigación, este estudio cuenta con varias limitaciones. Entre ellas, destaca que los resultados se obtienen de una muestra de conveniencia formada por participantes evaluados por profesionales y familiares que desearon participar, lo que limita el poder generalizar sus conclusiones. Tampoco se llevaron a cabo grupos de discusión con personas con TEA y familiares durante el desarrollo de la escala, puesto que se trató de adaptar una escala ya existente para personas con DI a aquellos que también presentan autismo. A pesar de ello, en el estudio Delphi para la creación de la versión piloto de la Escala KidsLife (Gómez et al., 2014) participaron varios profesionales e investigadores especializados en autismo, y durante el proceso de adaptación de la escala a TEA se consultó a varios expertos, quienes confirmaron la adecuación del contenido de la Escala KidsLife para este colectivo. Posteriormente, se comprobó que las propiedades psicométricas eran adecuadas en la población de estudio (Arias et al., 2018; Gómez, Morán, Alcedo, Arias et al., 2018). La tercera limitación hace referencia a que se trata de un informe completado por terceras personas. A pesar de que diversos estudios han encontrado una baja correlación entre los autoinformes de calidad de vida e informes de otras personas, estos últimos continúan siendo imprescindibles en el caso de las personas que presentan limitadas habilidades de comprensión y expresión para contestar de manera fiable por sus limitaciones en el funcionamiento intelectual o por su edad temprana. Es más, los informes de otras personas se consideran de gran utilidad en la obtención de información para el desarrollo y evaluación de la eficacia de intervenciones profesionales y estrategias organizacionales por ser más sensibles a los cambios. Así, siempre que sea posible, resulta recomendable combinar tales evaluaciones con autoinformes e implicar de forma activa a las personas con TEA en los procesos de evaluación y planificación (Vidriales, Cuesta, Hernández y Plaza, 2017).

Cabe destacar que este estudio es fruto del trabajo conjunto con los profesionales, familias y organizaciones, quienes demandaban contar con un instrumento de calidad de vida que tuviese en cuenta las posibles particularidades de las personas con TEA. En este sentido, la Escala KidsLife-TEA constituye la primera escala en el ámbito internacional de calidad de vida con contenido específicamente adaptado a niños y adolescentes con autismo, superando además sus propiedades psicométricas las de anteriores instrumentos de calidad de vida dirigidos a la infancia y la adolescencia en nuestro país. En el futuro, sería preciso examinar cuáles son las variables individuales y contextuales que tienen influencia en la calidad de vida de niños y jóvenes con TEA y Di y que podrían matizar los resultados presentados en este estudio preliminar. Además, se continuará con la adaptación y validación de la escala para otros países (p. ej., Países Bajos, Argentina, Colombia, Chile, Portugal) y se explorarán posibilidades de adaptar la Escala KidsLife para niños y jóvenes con TEA pero sin DI.

En conclusión, este estudio revela cómo áreas prioritarias de intervención la inclusión social y la autodeterminación. Más concretamente, parece de urgente necesidad la promoción de la participación en la vida comunitaria (p. ej., actividades de ocio y cultura, grupos deportivos, sociales, educativos) y en la toma de decisiones (p. ej., elaboración de su plan individual de apoyos, elegir la decoración de la habitación, la vestimenta, cómo y con quién pasar su tiempo libre) de los jóvenes con TEA y DI, con el fin último de mejorar su calidad de vida. 
INCLUSIÓN SOCIAL Y AUTODETERMINACIÓN: LOS RETOS EN LA CALIDAD DE VIDA DE LOS JÓVENES CON AUTISMO Y DISCAPACIDAD INTELECTUAL

M. ${ }^{a}$ LUCÍA MORÁN SUÁREZ, LAURA E. GÓMEZ SÁNCHEZ Y M. ${ }^{a}$ ÁNGELES ALCEDO RODRÍGUEZ

\section{Referencias bibliográficas}

Alcedo, M. A., Aguado, A. L., Arias, B., González, M. y Rozada, C. (2008). Escala de calidad de vida (ECV) para personas con discapacidad que envejecen: Estudio preliminar. Intervención Psicosocial, 17, 153-167.

Arias, B., Gómez, L. E., Verdugo, M. Á. y Navas, P. (2010). Evaluación de la calidad de vida en drogodependencias mediante el modelo de Rasch. Revista Española de Drogodependencias, 2, 206-219.

Arias, V. B., Gómez, L. E., Morán, M. L., Alcedo, M. A., Monsalve, A. y Fontanil, M. Y. (2018). Does quality of life differ for children with autism spectrum disorder and intellectual disability compared to peers without autism? Journal of Autism and Developmental Disorders, 48, 123-136. doi: 10.1007/s10803-017-3289-8.

Bigby, C., Knox, M., Beadle-Brown, J. y Bould, E. (2014). Identifying good group homes: Qualitative indicators using a quality of life framework. Intellectual Developmental Disabilities, 52 (5), 348-366. doi: 10.1352/1934-9556-52.5.348.

Burgess, A. F. y Gutstein, S. E. (2007). Quality of life for people with autism: Raising the standard for evaluating successful outcomes. Child and Adolescent Mental Health, 12 (2), 80-86. doi: 10.1111/j.1475-3588.2006.00432.x.

Carbó-Carreté, M., Guàrdia-Olmos, J., Giné, C. y Schalock, R. L. (2015). A structural equation model of the relationship between physical activity and quality of life. International Journal of Clinical and Health Psychology, 15, 236-252. doi: 10.1016/j.ijchp.2015.11.001.

Chiang, H. y Wineman, I. (2014). Factors associated with quality of life in individuals with autism spectrum disorders: A review of literature. Research in Autism Spectrum Disorders, 8, 974-986. doi: 10.1016/j.rasd.2014.05.003.

Chou, Y. C., Wehmeyer, M. L., Palmer, S. B. y Lee, J. (2016). Comparisons of self-determination among students with autism, intellectual disability, and learning disabilities: A multivariate analysis. Focus on Autism and Other Developmental Disabilities, 32 (2), 124-132. doi: $10.1177 / 1088357615625059$.

Chou, Y. C., Wehmeyer, M. L., Shogren, K. A., Palmer, S. B. y Lee, J. H. (2017). Autism and self-determination: factor analysis of two measures of self-determination. Focus on Autism and Other Developmental Disabilities, 32 (3), 163-175. doi: 10.1177/1088357615611391.

Claes, C., van Hove, G., Vandevelde, S., van Loon, J. y Schalock, R. L. (2012). The influence of supports strategies, environmental factors, and client characteristics on quality of life-related personal outcomes. Research in Developmental Disabilities, 33, 96-103. doi: 10.1016/j.ridd.2011.08.024.

Clark, B. G., Magill-Evans, J. E. y Koning, C. J. (2015). Youth with autism spectrum disorders: Self- and proxy-reported quality of life and adaptive functioning. Focus on Autism and Other Developmental Disabilities, 30, 57-64. doi: 10.1177/1088357614522289.

Cottenceau, H., Roux, S., Blanc, R., Lenoir, P., Bonnet-Brilhault, F. y Barthélémy, C. (2012). Quality of life of adolescents with autism spectrum disorders: Comparison to adolescents with diabetes. European Child and Adolescent Psychiatry, 21, 289-296. doi: $10.1007 / \mathrm{s} 00787-012-0263-\mathrm{z}$.

De VRIEs, M. y Geurts, H. M. (2015). Influence of autism traits and executive functioning on quality of life in children with an autism spectrum disorder. Journal of Autism and Developmental Disorders, 45 (9), 2734-2743. doi: 10.1007/s10803-015-2438-1.

Fernández, A., Morán, L., Campa, T., González, E., Gómez. L. E. y Monsalve, A. (2015). Resultados personales en autodeterminación en niños y adolescentes con discapacidad

(C) Ediciones Universidad de Salamanca / CC BY-NC-ND

Siglo Cero, vol. 50 (3), n. ${ }^{\circ}$ 271, 2019, julio-septiembre, pp. 29-46 
INCLUSIÓN SOCIAL Y AUTODETERMINACIÓN: LOS RETOS EN LA CALIDAD DE VIDA DE LOS JÓVENES CON AUTISMO Y DISCAPACIDAD INTELECTUAL

M. ${ }^{a}$ LUCÍA MORÁN SUÁREZ, LAURA E. GÓMEZ SÁNCHEZ Y M. ${ }^{a}$ ÁNGELES ALCEDO RODRÍGUEZ

intelectual y trastorno del espectro del autismo. En M. Á. VERDugo (Coord.), IX Jornadas cientificas de investigación sobre personas con discapacidad: Prácticas profesionales y organizacionales basadas en la evidencia [CD-ROM]. Salamanca: INICO.

Fernández, M., Gómez, L. E., Arias, V. B., Aguayo, V., Amor, A. M., Andelic, N., ... y Verdugo, M. Á. (2018). A new scale for measuring quality of life in acquired brain injury. Quality of Life Research. Manuscrito en revisión.

Fernández, M., Verdugo, M. Á., Gómez, L. E., Aguayo, V. y Arias, B. (2018). Core indicators to assess quality of life in population with brain injury. Social Indicators Research, 137 (2), 813-828. doi: 10.1007/s11205-017-1612-6.

Gómez, L. E. (2010). Evaluación de la calidad de vida en servicios sociales: Validación y calibración de la Escala GENCAT [Assessment of quality of life in social services: Validation and calibration of the Gencat Scale]. Tesis doctoral sin publicar. INIco, Universidad de Salamanca.

Gómez, L. E. (2014). Spanish social service recipients. En A. C. Michalos (Ed.), Encyclopedia of Quality of Life and Well-Being Research (pp. 6251-6258). Dordretch, Holanda: Springer.

Gómez, L. E., Alcedo, M. A., Arias, B., Fontanil, Y., Arias, V. B., Monsalve, M. A., ... y Verdugo, M. Á. (2016). A new scale for the measurement of quality of life in children with intellectual disability. Research in Intellectual and Developmental Disabilities, 53-54, 399-410. doi: 10.1016/j.ridd.2016.03.005.

Gómez, L. E., Alcedo, M. A., Verdugo, M. Á., Arias, B., Fontanil, Y., Arias, V. B, ... y Verdugo, M. Á. (2016). Escala KidsLife: Evaluación de la calidad de vida de niños y adolescentes con discapacidad intelectual. Salamanca: INIcO.

Gómez, L. E., Arias, B., Verdugo, M. Á. y Navas, P. (2012). An outcomes-based assessment of quality of life in social services. Social Indicators Research, 106, 81-93. doi: 10.1007/ s11205-011-9794-9.

Gómez, L. E., Arias, B., Verdugo, M. Á., Tassé, M. J. y Brown, I. (2015). Operationalisation of quality of life for adults with severe disabilities. Journal of Intellectual Disability Research, 59 (10), 925-941. doi: 10.1111/jir.12204.

Gómez, L. E., Morán, M. L., Alcedo, M. A., Arias, V. B., Navas, P. y Verdugo, M. Á. (2018). Addressing quality of life of children with autism spectrum disorders and intellectual disability. Manuscrito enviado para su publicación.

Gómez, L. E., Morán, L., Alcedo, M. A., Verdugo, M. Á., Arias, V. B., Fontanil, Y., ... y Monsalve, A. (2018). Escala KidsLife-TEA: Evaluación de la calidad de vida de niños y adolescentes con autismo y discapacidad intelectual. Salamanca: INICO.

Gómez, L. E., Peña, E., Alcedo, M. A., Monsalve, A., Fontanil, Y., Verdugo, M. Á., ... y Arias, B. (2014). El constructo de calidad de vida en niños y adolescentes con discapacidades múltiples y profundas: Propuesta para su evaluación. Siglo Cero, 45 (1), 56-69.

Gómez, L. E., Verdugo, M. Á. y Arias, B. (2015). Validity and reliability of the inicoFEAPS Scale: An assessment of quality of life for people with intellectual and developmental disabilities. Research in Intellectual and Developmental Disabilities, 36, 600-610. doi: 10.1016/j.ridd.2014.10.049.

Gómez, L. E., Verdugo, M. Á., Arias, B. y Arias, V. (2011). A comparison of alternative models of individual quality of life for social service recipients. Social Indicators Research, 101, 109-126. doi: 10.1007/s11205-010-9639-y.

Gómez, L. E., Verdugo, M. Á., Rodríguez, M., Arias, V. B., Morán, L., Arias, B., ... y Fontanil, Y. (2017). Escala KidsLife-Down: Evaluación de la calidad de vida de niños y adolescentes con sindrome de Down. Salamanca: INIco.

(C) Ediciones Universidad de Salamanca / CC BY-NC-ND

Siglo Cero, vol. 50 (3), n. ${ }^{\circ}$ 271, 2019, julio-septiembre, pp. 29-46 
INCLUSIÓN SOCIAL Y AUTODETERMINACIÓN: LOS RETOS EN LA CALIDAD DE VIDA DE LOS JÓVENES CON AUTISMO Y DISCAPACIDAD INTELECTUAL

M. ${ }^{a}$ LUCÍA MORÁN SUÁREZ, LAURA E. GÓMEZ SÁNCHEZ Y M. ${ }^{a}$ ÁNGELES ALCEDO RODRÍGUEZ

Gómez-Vela, M. y Verdugo, M. Á. (2009). Cuestionario de evaluación de la calidad de vida de alumnos adolescentes: manual de aplicación. Madrid: CEPE.

Idring, S., Lundberg, M., Sturm, H., Dalman, C., Gumpert, C. y Rai, D. (2015). Changes in prevalence of autism spectrum disorders in 2001-2011: Findings from the Stockholm youth cohort. Journal of Autism and Developmental Disorders, 45 (6), 1766-1773. doi: 10.1007/s10803-014-2336-y.

Ikeda, E., Hinckson, E. y Krägeloh, C. (2014). Assessment of quality of life in children and youth with autism spectrum disorder: A critical review. Quality of Life Research, 23, 10691085. doi: 10.1007/s11136-013-0591-6.

Jenaro, C., Verdugo, M. Á., Caballo, C., Balboni, G., Lachapelle, Y., Otbrebski, W., ... y ScHALOCK, R. L. (2005). Cross-cultural study of person-centered quality of life domains and indicators: A replication. Journal of Intellectual Disability Research, 49, 734-739. doi: 10.1111/j.1365-2788.2005.00742.x.

Knüppel, A., Jakobsen, H., Lauritsen, M. B. y Telléus, G. K. (2018). Psychometric properties of the INICO-FEAPs scale in a Danish sample with autism spectrum disorders. Research in Developmental Disabilities, 75, 11-21. doi: 10.1016/j.ridd.2018.01.013.

Kuhlthau, K., Kovacs, E., Hall, T., Clemmons, T., Orlich, F., Delahaye, J., ... y Sikora, D. (2013). Health-related quality of life for children with ASD: Associations with behavioral characteristics. Research in Autism Spectrum Disorders, 7, 1035-1042. doi: 10.1016/j. rasd.2013.04.006.

Ley 39/2006, de 14 de diciembre, de promoción de la autonomía personal y atención a las personas en situación de dependencia. Boletín Oficial del Estado, n 299, de 15 de diciembre de 2006, 44142-44156.

Luckasson, R., Borthwick-Duffy, S., Buntinx, W. H. E., Coulter, D. L., Craig, E. M., Reeve, A., ... y Tassé, M. J. (2002). Mental retardation: Definition, classification, and systems of supports (10. ${ }^{\mathrm{a}}$ ed.). Washington, DC: American Association on Mental Retardation.

Ministerio de Sanidad, Servicios Sociales e Igualdad (2015). Estrategia española en Trastornos del Espectro del Autismo. Recuperado de http://www.msssi.gob.es/ssi/discapacidad/.../estrategia_espanola_autismo.docx.

Morales-Hidalgo, P., Roige-Castellvi, J., Hernández-Martínez, C., Voltas, N. y CaNALS, J. (2018). Prevalence and characteristics of autism spectrum disorder among Spanish school-age children. Journal of Autism and Developmental Disorders, 48 (9), 3176-3190. doi: 10.1007/s10803-018-3581-2.

Morán, L., Gómez, L. E. y Alcedo, M. A. (2015). Relaciones interpersonales en niños y jóvenes con trastornos del espectro del autismo y discapacidad intelectual. Revista Española de Discapacidad, 3, 77-91. doi: 10.5569/2340-5104.03.01.04.

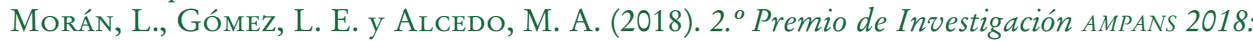
Hacia una mejor calidad de vida en jóvenes con discapacidad intelectual y autismo: Prioridades para la intervención y variables relevantes. Disponible en http://www.ampans.cat/ es/publicaciones-es/premio-de-investigacion.

Peralta, F. y Arellano, A. (2014). La autodeterminación de las personas con discapacidad intelectual: Situación actual en España. Revista CES Psicología, 7 (2), 59-77.

Sabeh, E., Verdugo, M. Á., Prieto, G. y Contini, N. (2009). CVI-CVip: cuestionario de evaluación de la calidad de vida en la infancia. Madrid: CEPE.

Santamaría, M., Verdugo, M. Á., Orgaz, B., Gómez, L. E. y Jordán de Urríes, B. (2012). Calidad de vida percibida por trabajadores con discapacidad intelectual en empleo ordinario. Siglo Cero, 43 (2), 46-61.

(C) Ediciones Universidad de Salamanca / CC BY-NC-ND

Siglo Cero, vol. 50 (3), n. ${ }^{\circ}$ 271, 2019, julio-septiembre, pp. 29-46 
INCLUSIÓN SOCIAL Y AUTODETERMINACIÓN: LOS RETOS EN LA CALIDAD DE VIDA DE LOS JÓVENES CON AUTISMO Y DISCAPACIDAD INTELECTUAL

M. ${ }^{a}$ LUCÍA MORÁN SUÁREZ, LAURA E. GÓMEZ SÁNCHEZ Y M. ${ }^{a}$ ÁNGELES ALCEDO RODRÍGUEZ

Schalock, R. L. (2015). Las mejores prácticas de las organizaciones y los profesionales. Siglo Cero, 46 (1), 7-23.

Schalock, R. L., Borthwick-Duffy, S. A., Bradley, V. J., Buntinx, W. H. E., Coulter, D. L., Craig, E. M., ... y Yeager, M. H. (2011). Discapacidad intelectual: Definición, clasifcación y sistemas de apoyo (11. ${ }^{\mathrm{a}}$ ed.). Madrid: Alianza Editorial.

Schalock, R. L., Keith, K. D., Verdugo, M. Á. y Gómez, L. E. (2010). Quality of life model development in the field of intellectual disability. En R. Koвer (Dir.), Quality of life for people with intellectual disability (pp. 17-32). Nueva York: Springer.

SCHAlOCK, R. L. y VeRdugo, M. Á. (2002/2003). Quality of life for human service practitioners. Washington, DC: American Association on Mental Retardation.

Schalock, R. L. y Verdugo, M. Á. (2012). A conceptual and measurement framework to guide policy development and systems change. Journal of Policy and Practice in Intellectual Disabilities, 7, 71-81. doi: 10.1111/j.1741-1130.2012.00329.x.

Schalock, R. L., Verdugo, M. Á., Gómez, L. E. y Reinders, H. (2016). Moving us towards a theory of individual quality of life. American Journal of Intellectual and Developmental Disabilities, 121 (1), 1-12. doi: 10.1352/1944-7558-121.1.1.

Tavernor, L., Barron, E., Rodgers, J., y McConachie, H. (2013). Finding out what matters: Validity of quality of life measurement in young people with ASD. Child, 39 (4), $592-$ 601. doi: 10.1111/j.1365-2214.2012.01377.x.

Van Heijst, B. F. C y Geurts, H. M. (2015). Quality of life in autism across the lifespan: A meta-analysis. Autism, 19, 158-167. doi: 10.1177/1362361313517053.

Van Loon, J. H., Bonham, G. S., Peterson, D. D., Schalock, R. L., Claes, C. y Decramer, A. E. (2013). The use of evidence based outcomes in systems and organizations providing services and supports to persons with intellectual disability. Evaluation and Program Planning, 36, 80-87. doi: 10.1016/j.evalprogplan.2012.08.002.

Van Loon, J., Claes, C. y Schalock, R. L. (2014). Improving quality of life, fulfilling rights: The use of the POS-A in support plans for people with ID. Journal of Applied Research in Intellectual Disabilities, 27 (4), 351.

Verdugo, M. Á., Arias, B., Gómez, L. E. y Schalock, R. L. (2010). Development of an objective instrument to assess quality of life in social services: Reliability and validity in Spain. International Journal of Clinical and Health Psychology, 10, 105-123.

Verdugo, M. Á., Gómez, L. E., Arias, B., Navas, P. y Schalock, R. L. (2014). Measuring quality of life in people with intellectual and multiple disabilities: Validation of the San Martín scale. Research in Developmental Disabilities, 35, 75-86. doi: 10.1016/j.ridd.2013.10.025.

Verdugo, M. Á., Gómez, L. E. y Navas, P. (2013). Discapacidad e inclusión: Derechos, apoyos y calidad de vida. En M. Á. Verdugo. y R. L. Schalock (Eds.), Discapacidad e inclusión, manual para la docencia (pp. 17-43). Salamanca: Amarú.

Verdugo, M. Á., Vicente, E., Fernández-Pulido, R., Gómez-Vela, M., Wehmeyer, M. L. y GuILlÉn, V. M. (2015). A psychometric evaluation of the ARC-INICO self-determination scale for adolescents with intellectual disabilities. International Journal of Clinical and Health Psychology, 15, 149-159. doi: 0.1016/j.ijchp.2015.03.001.

Vidriales, R., Cuesta, J. L., Hernández, C. y Plaza, M. (2017). Yo también decido. Guía práctica para que las personas con Trastorno del Espectro del Autismo y grandes necesidades de apoyo tomen decisiones sobre sus vidas. Madrid: Autismo España.

WANG, M., Schalock, R. L., Verdugo, M. Á. y Jenaro, C. (2010). Examining the factor structure and hierarchical nature of the quality of life construct. American Journal on Intellectual and Developmental Disabilities, 115 (3), 218-233. doi: 10.1352/1944-7558-115.3.218.

(C) Ediciones Universidad de Salamanca / CC BY-NC-ND

Siglo Cero, vol. 50 (3), n. ${ }^{\circ}$ 271, 2019, julio-septiembre, pp. 29-46 
INCLUSIÓN SOCIAL Y AUTODETERMINACIÓN: LOS RETOS EN LA CALIDAD DE VIDA DE LOS JÓVENES CON AUTISMO Y DISCAPACIDAD INTELECTUAL

M. ${ }^{a}$ LUCÍA MORÁN SUÁREZ, LAURA E. GÓMEZ SÁNCHEZ Y M. a ÁNGELES ALCEDO RODRÍGUEZ

White, K., Flanagan, T. D. y Nadig, A. (2018). Examining the relationship between selfdetermination and quality of life in young adults with autism spectrum disorder. Journal of Developmental and Physical Disabilities, 30 (6), 735-754. doi: 10.1007/s10882-018-9616-y.

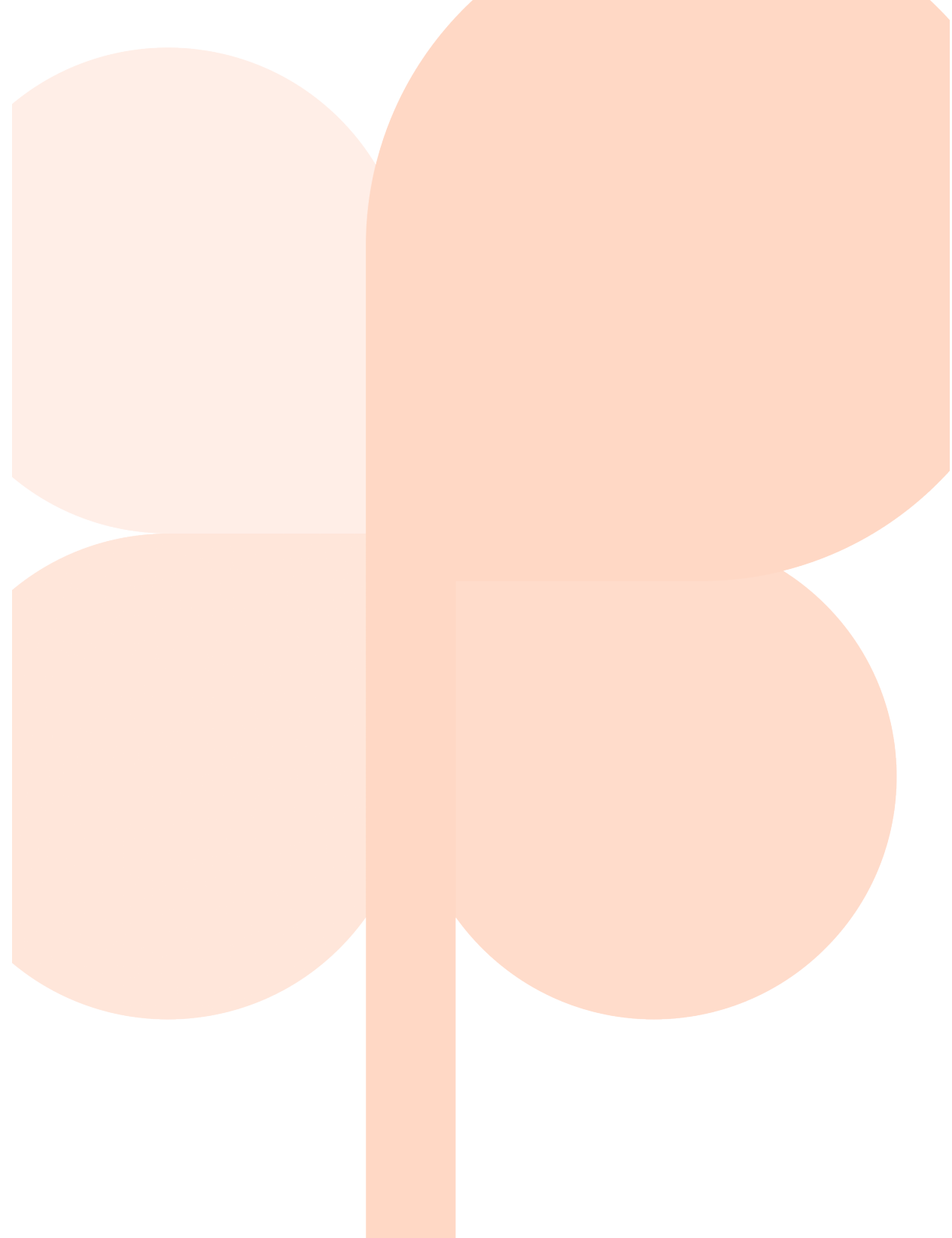

(C) Ediciones Universidad de Salamanca / CC BY-NC-ND

Siglo Cero, vol. 50 (3), n. ${ }^{\circ}$ 271, 2019, julio-septiembre, pp. 29-46 